\title{
Lymphoscintigraphic Mapping in Melanoma and Breast Cancer
}

\author{
Madelene A. Young \\ Cuyahoga Community College, Parma, Ohio
}

\begin{abstract}
CE credit: For CE credit, you can access the test for this article, as well as additional JNMT CE tests, online at https://www.snmmilearningcenter.org. Complete the test online no later than December 2023. Your online test will be scored immediately. You may make 3 attempts to pass the test and must answer $80 \%$ of the questions correctly to receive $1.0 \mathrm{CEH}$ (Continuing Education Hour) credit. SNMMI members will have their CEH credit added to their VOICE transcript automatically; nonmembers will be able to print out a CE certificate upon successfully completing the test. The online test is free to SNMMI members; nonmembers must pay $\$ 15.00$ by credit card when logging onto the website to take the test.
\end{abstract}

In determining the best options for lymphoscintigraphy, investigators have tried many different radiopharmaceuticals and protocols. There are varying opinions on the best route of administration, timing for delayed images, and radiopharmaceutical. Debate around which radiopharmaceutical is best arises from the requirement that it clear from healthy lymph channels quickly enough to reveal the SLN. This paper reviews and compares 9 recognized publications on lymphoscintigraphy, illustrates the different preferences regarding the procedure, and describes the generally accepted protocols for the best way to locate the sentinel lymph node. Detailed explanations are given for the variable involvement of the sentinel lymph node and the variations in disease stage seen with different protocols. All discrepancies between protocols are supported with evidence such as positive results or false-positive results. On reading this paper, you will be able to form an opinion on the best protocols and radiopharmaceuticals and learn why lymphoscintigraphy is a crucial tool in improving a patient's prognosis.

Key Words: sentinel lymph node; lymph node mapping; melanoma

J Nucl Med Technol 2020; 48:311-314

DOI: $10.2967 /$ jnmt.120.246918

$\mathbf{L}$

ymphoscintigraphy is used to track how malignant cells travel in lymph channels throughout the body. The lymph vessels that carry malignant cells follow patterns and trails yet are still unique to each type of malignancy. Different radiopharmaceuticals and techniques impact how adequately we can locate the sentinel lymph node (SLN).

\footnotetext{
Received Apr. 9, 2020; revision accepted Jul. 9, 2020.

For correspondence or reprints contact: Madelene A. Young, Department of Nuclear Medicine, Cuyahoga Community College, West Campus, Parma, $\mathrm{OH} 44130$.

E-mail: madeleneyoung108@gmail.com

Published online Sep. 4, 2020.

COPYRIGHT (c) 2020 by the Society of Nuclear Medicine and Molecular Imaging.
}

The SLN is the nodal site of potential micrometastasis. SLNs are the main focus of lymphoscintigraphy because these nodes indicate where malignant cells will spread first. This knowledge defines the route of potential treatment and the prognosis for melanoma patients. The presence of regional lymph node involvement is the single most important prognostic factor. Assessing how far the tracer has traveled also indicates how far the malignancy has traveled. SLNs are the first draining node in the lymphatic chain. These chains are normal free-flowing channels throughout the entire body. By identifying the SLN, or where the lymph fluid travels first in the body, one can map the route of the malignancy. The presence of an SLN on lymphoscintigraphy images does not indicate that a malignancy has been found. But by locating this node, surgeons can remove it and send a sample to be tested for malignancy.

Lymphoscintigraphy assesses obstruction, flow, and malignancy in lymph nodes. The theory of lymph node mapping entails injecting a radioactive tracer and performing imaging to follow the route of the tracer, determine where it builds up, and see how far it can travel. Lymphoscintigraphy relies on the SLN and lymphatic channels in the individual patient. There are thousands of cellular and physical differences between human beings. Malignancies that travel in lymph channels are among the most dangerous, and the exact placement of every SLN varies between individuals. Regionally, lymph nodes are typically found in standard areas from patient to patient. This standardized, regional flow gives the familiar patterns in which SLNs are commonly found; however, discovery of abnormal SLNs is not unusual.

\section{CLASSIFICATION OF MELANOMA}

Melanoma, a common cancer, begins in the pigmented (dark) cells in the skin called melanocytes. More than 70,000 patients are diagnosed with-and over 9,000 die from-melanoma yearly (1). Malignant melanoma is classified as superficial spreading, nodular, lentigo maligna, or acral lentiginous (1). In Table 1 these subtypes are shown according to their growth patterns and potential for spread. 
TABLE 1

Differences in Melanoma Subtypes, Their Impact on the Body, and Who Is Most at Risk

\begin{tabular}{|c|c|c|c|c|c|}
\hline Subtype & Incidence & Location & Growth pattern & Metastasis potential & Sex most at risk \\
\hline Superficial spreading & $70 \%$ & $\begin{array}{l}\text { Non-weight-bearing } \\
\text { surfaces of foot }\end{array}$ & Slow and radial & Low & Women \\
\hline Nodular & $15 \%-30 \%$ & $\begin{array}{l}\text { Non-weight-bearing } \\
\text { surfaces of foot }\end{array}$ & Rapid and vertical & High & Men \\
\hline Lentigo maligna & $4 \%-10 \%$ & $\begin{array}{l}\text { Anterior lower leg in } \\
\text { women; head and } \\
\text { trunk in men }\end{array}$ & Slow and radial & Low & Women \\
\hline Acral lentiginous & $2 \%-8 \%$ & Palms, soles, nail beds & $\begin{array}{l}\text { Radial at first, } \\
\text { then vertical }\end{array}$ & High & Women \\
\hline
\end{tabular}

Each subtype follows a different metastatic spreading pattern.

\section{MELANOMA IMAGING TECHNIQUES}

Many variables impact the quality of lymphoscintigraphic findings for melanoma. Its location and type, preparation of the radiopharmaceutical, injection technique, and image views are crucial to the quality and consistency of the findings. The radiopharmaceutical injection relies on the lymphatic system's ability to transport large molecules from the interstitial spaces back to the vascular system (2). Differences in molecule size present different challenges, such as lymphatic response time. Responses that are too quick cannot be captured on a nuclear image. Responses that are too slow make it difficult to find an SLN.

The ideal imaging agents for lymphoscintigraphy of cutaneous melanoma clear rapidly from the injection site into the lymphatic system, expose the patient to only a low radiation dose, are retained well within the lymph nodes, and have the correct particle size (depending on targeted findings).

Two main types of tracers are used: macromolecules and colloidal suspensions. Small particles $(<100 \mathrm{~nm})$ clear from the interstitial spaces, whereas larger particles $(>500 \mathrm{~nm})$ tend to remain at the injection site (3). ${ }^{99 \mathrm{~m}} \mathrm{Tc}$-labeled colloids clear more slowly from the injection site than macromolecules and do not show lymphatic vessels in great detail (4). However, colloidal suspensions are trapped much more effectively in lymph nodes, making them more suitable for lymph node mapping (4). Because the amount of radioactivity injected into the patient is microscopic $(<37 \mathrm{MBq}[1 \mathrm{mCi}])$, the radiation dose to the patient is often considered insignificant (5). Regardless of the tracer used, the injection technique is crucial. Injection of too large a quantity may slow uptake of the radiopharmaceutical and contaminate the patient's skin. Different tracers have different uptake times. Uptake time is also impacted by the quality of the injection.

The standardized protocol of the Society of Nuclear Medicine and Molecular Imaging for melanoma lymphoscintigraphy (6) uses ${ }^{99 \mathrm{~m}} \mathrm{Tc}$-labeled filtered sulfur colloid or ${ }^{99 \mathrm{~m} T c-t i l m a n o c e p t ~(L y m p h o s e e k ; ~ C a r d i n a l ~ H e a l t h) . ~ T h e ~}$ target dose is $15 \mathrm{MBq}(0.5 \mathrm{mCi})$, administered intradermally or subcutaneously. The dose is divided equally among 4 syringes; these 4 injections surround the lesion. The acquisition parameters depend on the region of interest (ROI). The camera is placed over the ROI and acquires a dynamic image for 30-60 min (6). Static images, in both axillary and inguinal views, of the ROI and of any visualized lymph nodes are taken for 5-10 min. Images of the head and neck should be both anterior and posterior. Additional views, such as oblique, can be acquired as needed, or SPECT images can be obtained. The location of the SLN is marked on the skin. It is not advisable to acquire images at more than $15 \mathrm{~h}$ after injection (6). Figure 1 shows an example of lymph node localization in the groin; the melanoma is in the thigh region.

Additional options in the standardized protocol include using a transmission source, such as ${ }^{57} \mathrm{Co}$, to delineate the body contour of the patient. Radioactive markers may also be used as reference points to provide more anatomic information. Anatomic reference points are subject to interpretation; however, when a transmission source is included, the body contour is illuminated. The effect is similar to that of a SPECT image, in that it provides more than just counts per image; the contour of the body is visible and can help guide interpretations. Usually, patients undergo surgery after lymphoscintigraphy procedures. This transmission-source body-contour image can be referenced by the surgeon to confirm the target lymph node.

\section{CLASSIFICATION OF BREAST LESIONS}

Malignant breast lesions are classified into 5 groups. (BCoding System; established by the NHS Breast Cancer Screening Program). B1 is normal tissue that does not require

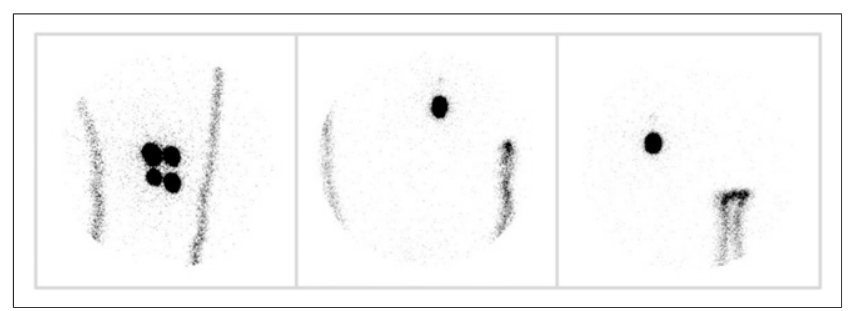

FIGURE 1. Leg melanoma with visualized node in groin. This image shows localization of SLN in groin area when injection is performed at thigh. Image provides idea of generalized regions in which lymph nodes are frequently found. 
further diagnostic testing. B2 is benign lesions that have been biopsied and have completed diagnostic testing. B3 is lesions with potential for malignancy, including papillary lesions, scar tissue, and classic lobular neoplasia; biopsy is necessary for B3 lesions to determine further action. B4 is malignancy, or invasive carcinoma, that has been confirmed through biopsy and diagnostic imaging. B5 lesions are malignant in situ, invasive and employ unequivocal malignant process; additional organs and systems are often involved when B5 lesions are confirmed.

\section{BREAST IMAGING TECHNIQUES}

Similar to lymphoscintigraphy imaging of melanoma, lymphoscintigraphy imaging of the breast uses many different protocols, which lead to differing results. The radiopharmaceuticals used are the same as for melanoma lymphoscintigraphy: ${ }^{99 \mathrm{~m}}$ Tc-labeled filtered sulfur colloid or ${ }^{99 \mathrm{~m}}$ Tc-tilmanocept. The dose administered is also about the same, ranging from $15 \mathrm{MBq}(0.5 \mathrm{mCi})$ to $35 \mathrm{MBq}(1.0 \mathrm{mCi})$. Injections should be subareolar or peritumoral, surrounding the ROI. In the standardized protocol of the Society of Nuclear Medicine and Molecular Imaging (7), the acquisition includes a 30-min dynamic image and static images from 10-15 min per view. The tracer is to be administered $0.25-3 \mathrm{~h}$ before surgery, and no imaging should be performed $15 \mathrm{~h}$ post injection (8).

The imaging findings are usually confined to the axillary area. Figure 2 illustrates images showing an SLN in the axilla. The depth of the lymph node is what makes this study more difficult to read. Even if SLNs are clearly seen to be in the axillary region, the reading physician cannot pinpoint the lymph node for excision if the body contour is not visible. Figure 3 illustrates images that were obtained using a transmission source and show the body contour.

\section{DOWNSIDES TO LYMPHOSCINTIGRAPHY}

Although lymphoscintigraphy clearly has many uses and benefits, such as tracking cancer before it has spread beyond borders, the lack of a standardized protocol hinders its full potential. Since lymphatics are unpredictable, it is imperative that the same protocol be used when lymphoscintigraphy is repeated in a patient. Postoperative scans are done at the 3- to 6-mo mark. If the same protocol is

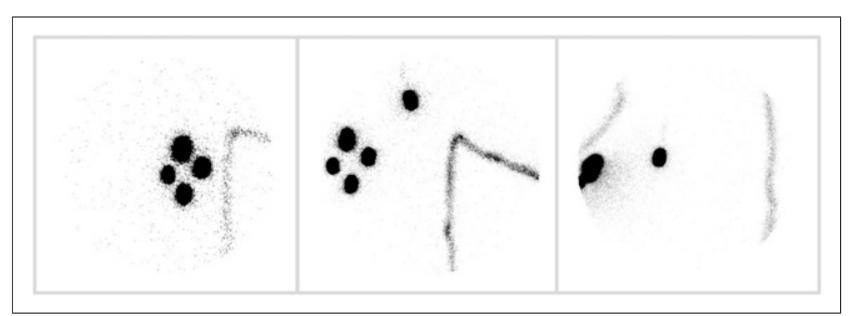

FIGURE 2. Breast lesion with visualized lymph node in axilla. Compare with Figure 3 to see benefits of using transmission source when imaging SLNs. followed in preoperative and postoperative scans, $85 \%$ of patients have consistency between the two, identifying the same SLN. Differing protocols may lead to false identification or a missed malignant node.

The definition of an abnormality also varies among institutions, thus affecting staging, treatment plan, and prognosis. If images are taken at $0,30,120$, and $240 \mathrm{~min}$ at one institution but at 0,60 , and $240 \mathrm{~min}$ at another location (both protocols are widely used), comparison between patient images will be misleading. The radiopharmaceuticals used should also be standardized. If one institution scans with smaller particles and another with larger particles, the smaller particles will appear to travel farther than the larger ones. Use of the wrong particle size can give a false-negative if large particles remain at the injection site, leading physicians to believe the malignant cells are not likely to spread.

Nissenkorn compared the use of whole-body lymphoscintigraphy, lymphangiography, and CT to evaluate for metastasis. A correct diagnosis was made in $61.5 \%$ of patients, whereas $23.1 \%$ had false-positive results. False-positive results were twice as common for lymphoscintigraphy as for lymphangiography and CT (9). This result demonstrates that even within the same health-care facility, lymphoscintigraphy procedures can be inconsistent.

The standardization of lymphoscintigraphy protocols will advance this modality to the point that it may surpass other modalities. If physicians can compare studies that are performed according to the same protocol worldwide, more patients will receive accurate treatment and preventative measures.

\section{PRACTICAL USES FOR LYMPHOSCINTIGRAPHY}

Lymphoscintigraphy is also a preoperative tool. The images can identify and localize lymph node involvement before nodovenous shunt procedures, SLN biopsies, and lymph node dissection. SLN biopsy is the most commonly used procedure in conjunction with lymphoscintigraphy. Early-stage abnormalities may also be detected in unsuspected areas when comparing the whole-body images with specific regions defined by lymphoscintigraphy images.

Lymphedema patients are also the subject of a large percentage of lymphoscintigraphy studies. Assessment of lymphatic drainage pinpoints where the channels are not draining properly. This malfunction causes a buildup of fluid, which is often painful. Lymphedema is very treatable or can be managed to a functional state once the ROI is defined (8). Knowledge of the ROI through lymphoscintigraphy can be used to repair specific sites rather than treating the entire affected area.

Imaging technique is also crucial, considering the views taken are to be compared before and after therapy or surgery. Lymphoscintigraphy has improved the accuracy of melanoma staging using SPECT/CT and PET/CT. Many of the lymphatic drainage routes assessed by lymphoscintigraphy and SPECT/CT correspond to sites of lymph node metastases. The acquisitions differ in timing, position, and field of view. 


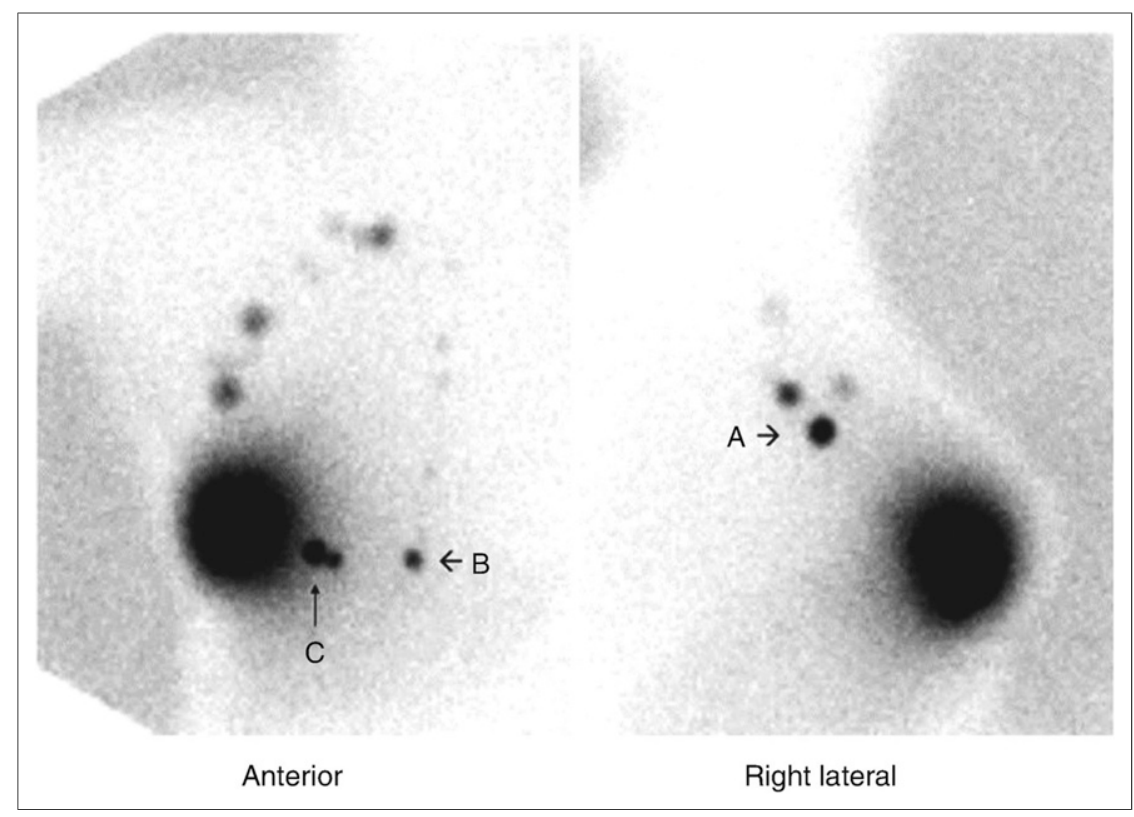

FIGURE 3. Breast lesion lymphoscintigraphy with ${ }^{57} \mathrm{Co}$ transmission source (7). This image shows how transmission source can be effective tool when trying to relate internal node to external site on body. By showing body outline, image makes it easier to mark node for surgery. $A=S L N$ from lateral view; $B$ and $C=$ other significant nodes, from anterior view, in ROI. These nodes may also be removed for biopsy.

Flood sources are used to define the body contour, allowing depiction of more centrally located lymph nodes and delineation of the nodes from the lymphatic channels.

Internationally, lymphoscintigraphy is being performed at thousands of institutions using hundreds of protocols. The resulting inconsistency between images makes comparisons unreliable. Standardizing the protocol for such a widely used imaging modality would enable physicians to consult similar cases when diagnosing and treating melanoma patients.

\section{THE FUTURE POTENTIAL OF LYMPHOSCINTIGRAPHY}

The many protocols used in lymphoscintigraphy, and the vast diversity among patients, affect outcomes. Standardizing and refining protocols among institutions that share images would allow for more accurate diagnoses. Lymphoscintigraphy is a widely available modality that has the capacity to embrace the use of other radiopharmaceuticals.
For example, although the use of ${ }^{99 \mathrm{~m}} \mathrm{Tc}$-labeled filtered sulfur colloid allows tracking of melanoma and breast cancer, the use of a different tracer might enable tracking of other types of malignancy. The field of lymphoscintigraphy is advancing every day, yet inconsistency in protocols may hinder its future potential.

\section{DISCLOSURE}

No potential conflict of interest relevant to this article was reported.

\section{ACKNOWLEDGMENT}

I thank Catherine Bloor, BS, RT $(\mathrm{N})$, and James Fisher BS, CNMT, $\mathrm{RSO}$, for supporting this research.

\section{REFERENCES}

1. Weiss J, Bertz J, Jung EG. Malignant melanoma in southern Germany: different predictive value of risk factors for melanoma subtypes. Dermatologica. 1991;183:109-113.

2. Yudd AP, Kempf JS, Goydos JS, Stahl TJ, Feinstein RS Use of sentinel node lymphoscintigraphy in malignant melanoma. Radiographics. 1999;19:343-353.

3. Perissinotti A, Rietbergen DD, Vidal-Sicart S, Riera AA, Olmos RAV. Melanoma \& nuclear medicine: new insights \& advances. Melanoma Manag. 2018;5:MMT06.

4. Melanoma. SNMMI website. http://www.snmmi.org/AboutSNMMI/Content.aspx? ItemNumber=995. Accessed September 28, 2020.

5. Kaplan WD, Davis MA, Rose CM. A comparison of two technetium-99m-labeled radiopharmaceuticals for lymphoscintigraphy: concise communication. $\mathrm{J} \mathrm{Nucl}$ Med. 1979;20:933-937.

6. Alazraki N, Glass EC, Castronovo F, Olmos RA, Podoloff D; Society of Nuclear Medicine. Procedure guideline for lymphoscintigraphy and the use of intraoperative gamma probe for sentinel lymph node localization in melanoma of intermediate thickness 1.0. J Nucl Med. 2002;43:1414-1418.

7. Giammarile F, Alazraki N, Aarsvold J; Society of Nuclear Medicine. The EANM and SNMMI practice guideline for lymphoscintigraphy and sentinel node localization in breast cancer. Eur J Nucl Med Mol Imaging. 2013; 40:1932-1947.

8. Kalawat TC, Chittoria RK, Reddy PK, Suneetha B, Narayan R, Ravi P. Role of lymphoscintigraphy in diagnosis and management of patients with leg swelling of unclear etiology. Indian J Nucl Med. 2012;27:226-230.

9. Nissenkorn I, Winkler H, Servadio C, et al. A comparative evaluation of lymphoscintigraphy versus lymphangiography and computerized tomography scanning in diagnosis of lymph node metastases in advanced bladder cancer. J Urol. 1986;136: $825-827$. 